\title{
Selecting stimulation intensity in repetitive transcranial magnetic stimulation studies: A systematic review between 1991 and 2020
}

\author{
Zsolt Turi $^{1,2}$ (D) | Maximilian Lenz ${ }^{1}$ ｜ Walter Paulus ${ }^{2} \quad$ Matthias Mittner $^{3}$ (iD | \\ Andreas Vlachos ${ }^{1,4,5}$
}

${ }^{1}$ Department of Neuroanatomy, Institute of Anatomy and Cell Biology, Faculty of Medicine, University of Freiburg, Freiburg, Germany

${ }^{2}$ Department of Clinical Neurophysiology, University Medical Center Göttingen, Göttingen, Germany

${ }^{3}$ Department of Psychology, UiT - The Arctic University of Norway, Tromso, Norway

${ }^{4}$ Center Brain Links Brain Tools, University of Freiburg, Freiburg, Germany

${ }^{5}$ Center for Basics in Neuromodulation, Faculty of Medicine, University of Freiburg, Freiburg, Germany

\section{Correspondence}

Zsolt Turi, Department of Neuroanatomy, Institute of Anatomy and Cell Biology,

Faculty of Medicine, University of Freiburg, Freiburg, Germany.

Email: zsolt.turi@anat.uni-freiburg.de

\section{Funding information}

NIH, Grant/Award Number:

1R01NS109498-01A1; University Medical Center Göttingen; Albert-Ludwigs-

Universitaet Freiburg

\begin{abstract}
Repetitive transcranial magnetic stimulation (rTMS) is an increasingly used, noninvasive brain stimulation technique in neuroscience research and clinical practice with a broad spectrum of suggested applications. Among other parameters, the choice of stimulus intensity and intracranial electric field strength substantially impacts rTMS outcome. This review provides a systematic overview of the intensity selection approaches and stimulation intensities used in human rTMS studies. We also examined whether studies report sufficient information to reproduce stimulus intensities for basic science research models. We performed a systematic review by focusing on original studies published between 1991 and 2020. We included conventional (e.g., 1 or $10 \mathrm{~Hz}$ ) and patterned protocols (e.g., continuous or intermittent theta burst stimulation). We identified 3,784 articles in total, and we manually processed a representative portion (20\%) of randomly selected articles. The majority of the analyzed studies (90\% of entries) used the motor threshold (MT) approach and stimulation intensities from $80 \%$ to $120 \%$ of the MT. For continuous and intermittent theta burst stimulation, the most frequent stimulation intensity was $80 \%$ of the active MT. Most studies (92\% of entries) did not report sufficient information to reproduce the stimulation intensity. Only a minority of studies (1.03\% of entries) estimated the rTMS-induced electric field strengths. We formulate easy-to-follow recommendations to help scientists and clinicians report relevant information on stimulation intensity. Future standardized reporting guidelines may facilitate the use of basic science approaches aiming at better understanding the molecular, cellular, and neuronal mechanisms of rTMS.
\end{abstract}

\section{K E Y W O R D S}

continuous theta burst stimulation, intermittent theta burst stimulation, repetitive transcranial magnetic stimulation, reproducibility, stimulation intensity, transparency

\footnotetext{
Abbreviations: cTBS, continuous theta burst stimulation; FDA, Food and Drug Administration; iTBS, intermittent theta burst stimulation; MRI, magnetic resonance imaging; MT, motor threshold; PRISMA, Preferred Reporting Items for Systematic Reviews and Meta Analyses; rTMS, repetitive transcranial magnetic stimulation.

Edited by: Gregor Thut

Matthias Mittner and Andreas Vlachos shared senior authorship.

This is an open access article under the terms of the Creative Commons Attribution NonCommercial License, which permits use, distribution and reproduction in any medium, provided the original work is properly cited and is not used for commercial purposes.

() 2021 The Authors. European Journal of Neuroscience published by Federation of European Neuroscience Societies and John Wiley \& Sons Ltd.
} 


\section{INTRODUCTION}

Repetitive transcranial magnetic stimulation (rTMS) noninvasively induces electromagnetic fields in the brain (Saturnino et al., 2019). It is increasingly applied in neuroscience research and clinical treatment (Blumberger et al., 2018; Suppa et al., 2016). The efficacy of rTMS, besides the state of the receiving brain, significantly depends on the stimulation parameters, collectively referred to as the dose.

Based on the definition of Peterchev and colleagues (Peterchev et al., 2012), rTMS dose refers to all participantindependent device parameters that affect the spatial and temporal characteristics of the electromagnetic field produced in the brain. These include defining both the user-adjustable parameters (e.g., the percentage of maximum pulse intensity) and non-adjustable parameters (e.g., coil inductance) (Peterchev et al., 2012). In this definition, the term "independent" refers to the notion that all stimulation parameters are translated into physical, participant-independent device parameters (Peterchev et al., 2012).

Selecting the stimulation intensity is an important step in determining rTMS dose. There are several intensity selection approaches, and researchers, as well as clinicians, may face two obvious challenges in this process. The first is conceptual: How to determine the stimulation intensity prospectively, such that the application of rTMS leads to the desired effects? The second is practical: For the sake of reproducibility, which parameters are crucial to standardize across experiments, and what information must be reported for a given protocol?

The goal of the present systematic review was to provide a comprehensive overview of the different intensity selection approaches and stimulation frequencies. To this aim, we performed a careful literature search to identify the different approaches and quantify their relative frequency of use. Moreover, we evaluated whether studies report sufficient information required to reproduce the stimulation intensity. Reporting the stimulation parameters in a reproducible manner is essential for research and clinical applications (Peterchev et al., 2012). Indeed, standardized reporting guidelines are crucial to informing computational and basic science research approaches to investigate the cellular, molecular, and network mechanisms of clinically relevant rTMS protocols (e.g., Lenz et al., 2016). We formulate easy-to-follow recommendations to help improve the reporting quality of rTMS studies.

\section{$2 \mid$ METHODS}

\section{1 | Research integrity}

The data, the code for the literature search, and the reported analyses are available for download at the following repository: https://github.com/ZsoltTuri/2021_rTMS_inten sity_review_paper.

\subsection{Creating the database}

A systematic literature search was performed by identifying rTMS studies to create a comprehensive database. The goal was to provide a systematic overview of the different intensity selection approaches and stimulation frequencies. We searched for articles that were published between January 1, 1991, and July 31, 2020. To this aim, we used $\mathrm{R}$ (version 4.0.2) (R Core Team, 2020), RStudio integrated development environment (version 1.3.1073) (RStudio Team, 2020), and the RISmed library (version 2.1.7) (Kovalchik, 2017).

We performed the literature search on the PubMed engine that mainly accesses the MEDLINE database. The RISmed $\mathrm{R}$ library was used to algorithmically perform the literature search. PubMed lists more than 30 million citations (source from https://en.wikipedia.org/wiki/PubMed; retrieved on January 19, 2021) and the search results are independent from the institutional subscription. The following search terms were used: 'repetitive transcranial magnetic stimulation', 'rTMS', 'repetitive AND TMS', 'rhythmic AND TMS', 'theta burst stimulation', 'theta burst AND stimulation', 'cTBS', and 'iTBS'. For each search term, we used the 'all fields' search field description tag.

The search revealed 6,727 PubMed hits in total, and we manually screened them to identify peer-reviewed original studies that applied rTMS on humans. The manual screening was performed on the title, abstract, and full text. We identified 3,804 articles that matched the criteria, and we could gain access to 3,784 articles. The remaining articles belonged to (a) other article types (e.g., reviews, editorials, commentaries, letters, meta-analyses, video-audio media, published erratum, retraction of publications), (b) other stimulation types (e.g., single-pulse TMS, pairedpulse TMS, peripheral application of repetitive magnetic stimulation that incorrectly used the term transcranial, transcranial magnetic seizure therapy, transcranial static magnetic stimulation, rotating transcranial magnetic stimulation technologies, other electrical stimulation techniques), (c) animal studies, (d) in vitro studies, or (e) the article was not available in English. We applied no further criteria for selecting the articles. Therefore, we included all rTMS articles irrespective of their stimulation parameters, sample characteristics or outcome measures. Note that we did not follow the Preferred Reporting Items for Systematic Reviews and Meta Analyses (PRISMA) guidelines in this study and hence, we did not register the evaluation protocol. 


\subsection{Randomly sampling from the database}

Two independent random samples were taken from the 3,784 accessed articles using a multi-step procedure implemented algorithmically in R. The goal of processing two independent samples was to cross-validate results found in one sample with results from the second one and to assess estimation error. Since the number of articles depends on the year of publication, we used year-dependent, stratified sampling. In each step, we sampled and processed 380 articles, which corresponds to $\sim 10 \%$ of the total number of articles (see Table S1).

In the first sampling step, we selected all articles between 1991 and 1998, because in these early years we identified only a few articles $(n=41)$. In 1999 , we randomly took 14 articles and between 2000 and 2010, we randomly picked 15 articles in each year $(n=165)$, because there were $<200$ publications per year. Between 2011 and 2020, we randomly selected 16 articles ( $n=160$; see Table $\mathrm{S} 1)$.

In the second sampling step, we selected 13 articles for the year 1999, since we identified only 27 articles in this year. Moreover, we randomly picked 15 articles in each year between 2000 and 2010 ( $n=165$ articles), 20 articles between 2011 and $2019(n=180)$ and 22 articles for year 2020 (see Table S1). We selected the number of random samples to ensure that each independent sample yields the same total number of articles (i.e., 380).

After the second step, we compared the result with the combined results of the first and second steps. We assessed similarity and compared the results from the steps based on the stimulation intensity selection approaches. If the results were not different, we interpreted the findings to indicate that the results could be generalized to the entire database.

In case of a discrepancy, we planned to continue with the next step until we observed no difference in the results between the last and second-last steps. However, this was not necessary, because we observed highly similar results between the first and second sampling (see Figure S1). Thus, we processed 760 articles, which corresponds to $\sim 20 \%$ of the total number of articles.

\subsection{Extracting the stimulation parameters}

We performed a text-mining search to identify the stimulation intensity approaches, the stimulation intensity, and device-specific information using R library pdfsearch (version 0.3.0) (LeBeau, 2018) on the 760 randomly selected articles. The keyword search was performed on full text. Moreover, we also evaluated on the entire database, whether studies report the electric field values (see Table S2 for details). Note that we only used the text-mining search to facilitate the manual information extraction process; all information for each study was manually assessed on full text.

Only real stimulation protocols were considered. Consequently, entries from sham/control protocols were not included in this review. Because this review focused on how rTMS studies determine the stimulation intensity and its relationship to the stimulation frequency, we created a new entry for every unique combination of the stimulation frequency and intensity in a given study. For instance, we created two separate entries for the scenario, when a given study used $1 \mathrm{~Hz}$ rTMS at $90 \%$ and $110 \%$ of the resting motor threshold (MT).

However, studies may also manipulate other stimulation parameters, such as the stimulation location, stimulation duration (number of pulses), inter-train intervals, stimulation timing, number of rTMS sessions or medications combined with rTMS. In case of additional stimulation parameters, we added new entries up to three values. For example, if a mapping study tested the 1 and $10 \mathrm{~Hz}$ protocols in six different locations for each frequency, we added only two new entries to the database (corresponding to the two frequencies). Note that despite adding new entries, we did not code these additional parameters (e.g., stimulation location, stimulation duration, etc.) separately in the database. We found 29 entries with multiple sites, one entry with multiple pulse numbers, six entries with multiple sessions, and three multiple medication conditions.

\section{3 | RESULTS}

\subsection{Intensity selection approaches}

The number of original studies steadily increased during the observation period, reaching 250-300 publications per year during the past 5 years (see Figure 1). We processed 760 randomly sampled articles, identified 241 unique protocols and added 1,378 entries in total. Because the details of the stimulation intensity parameters may differ between the entries in a given study, we decided to report the number of entries, not studies, unless stated otherwise. Consequently, the associated percentage values refer to the percentage of the total number of identified entries.

We identified three main stimulation intensity selection approaches: (a) threshold based ( $n=1,257 ; 91.22 \%)$, (b) fixed intensity $(n=91 ; 6.6 \%)$, and (c) electric field estimation based $(n=2 ; 0.15 \%)$. A small portion of entries failed to report the approach $(n=28 ; 2.03 \%)$. For an overview, see Figure 2.

The most common threshold-based approach was the MT approach $(n=1,232 ; 89.40 \%)$. Most studies used the resting threshold $(n=683 ; 49.56 \%)$ or the active MT approach $(n=233 ; 16.91 \%)$. Some studies did not specify the type of 


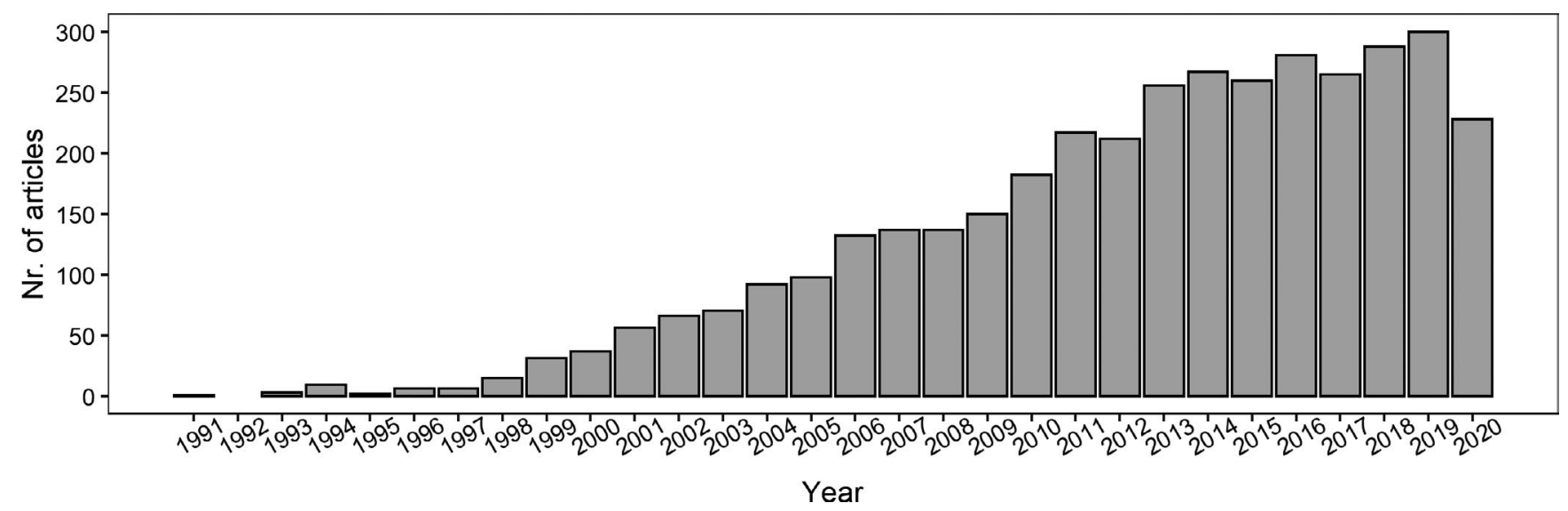

F I G U RE 1 Number of identified original studies performing rTMS on humans. The number of articles per year is shown between January 1 , 1991 and July 31,2020

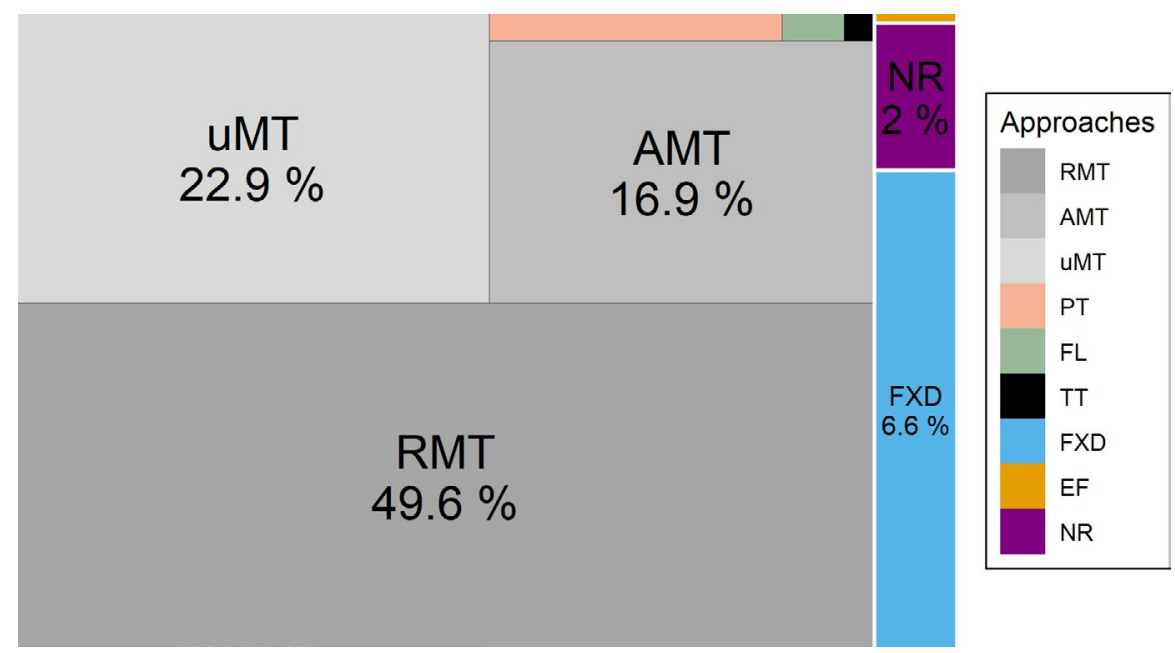

F I G URE 2 Stimulation intensity selection approaches and their relative frequency of use, by percentage of the total number of identified entries $(n=1,378)$. The sizes of rectangles are proportional to the percentage values. AMT indicates active motor threshold; EF, electric-fieldbased intensity selection; FL, functional lesion threshold; FXD, fixed intensity; NR, not reported; PT, phosphene threshold; RMT, resting motor threshold; TT, tolerability threshold; uMT, unspecified motor threshold

threshold $(n=316 ; 22.93 \%)$. Throughout the article, we use the abbreviation MT to collectively refer to the active, resting, and unspecified MT. Otherwise, we use the adjectives active, resting, or unspecified to explicitly refer to a given subtype of the MT.

Most studies used the method of limit $(n=776$, $56.31 \%$ ) to estimate the MT. In this approach, the minimal device output is determined by decreasing or increasing the device output required to produce motor-evoked potentials with a minimum predefined peak-to-peak amplitude $50 \%$ of the time in a given number of trials (Borckardt et al., 2006). Some studies did not specify the exact method; instead, they referred to previous publications via citation ( $n=107,7.76 \%)$. A small subset of studies used other algorithms to estimate the MT (e.g., parameter estimation by sequential testing methods) (Awiszus, 2014;
Borckardt et al., 2006) $(n=28,2.03 \%)$. Many studies did not report any details of the method used to estimate the MT $(n=340,24.67 \%)$.

In some studies, the MT was determined by recording motor-evoked potentials with surface electrodes $(n=705$, $51.16 \%)$. In other studies, MT was visually identified by observing the muscle twitches ( $n=177,12.84 \%)$. In many cases, the authors provided no information about how the MT was detected $(n=350,25.40 \%)$.

Some studies used additional, although less common, threshold-based approaches. A small number of studies used the phosphene threshold $(n=19,1.38 \%)$, the threshold for inducing "functional lesions" $(n=4,0.29 \%)$, such as speech arrest, or the tolerability threshold (i.e., the maximum intensity of comfortable stimulation; $n=2,0.15 \%$ ) to set the stimulation intensity. 


\subsection{Most studies use the MT approach at $80 \%-120 \%$ of the threshold}

The most frequent intensity selection approach was the MTbased one. Here, the stimulation intensity is typically expressed as the percentage (e.g., 90\%) of the MT. Two methods are commonly used for estimating the MT. The active MT requires the slight contraction of the target muscle by $\sim 10 \%-20 \%$ of the maximum contraction intensity. The resting MT is assessed during the complete relaxation of the target muscle.

We selected articles that (a) used the MT approach, (b) used a single stimulation intensity, and (c) reported the stimulation intensity and frequency $(n=1,162,84.33 \%)$. Of these studies, the stimulation intensity typically ranged from $80 \%$ to $120 \%$ of the MT (Figure 3). Studies using resting MT were the most frequent (Figure 3b).

\section{3 | Stimulation intensity as a function of stimulation frequency}

Next, we focused on the relationship between the stimulation intensity and stimulation frequency. We used the same selection criteria described in the previous point.

We divided the rTMS protocols into conventional and patterned ones. Conventional protocols use single frequencies (e.g., $1 \mathrm{~Hz}$ ) (Chen et al., 1997), whereas patterned protocols combine at least two stimulation frequencies (e.g., 5 and $50 \mathrm{~Hz}$ as in theta burst rTMS) (Huang et al., 2005). Moreover, patterned protocols use standardized stimulation parameters, such as inter burst intervals. We also identified studies using quadripulse protocols that deliver four monophasic pulses with interpulse intervals of 5 or $50 \mathrm{~ms}$ (Hamada et al., 2007; Matsumoto \& Ugawa, 2020). However, due to the low occurrence rate in our sample $(n=5,0.36 \%)$, we did not analyze these protocols in detail.

Focusing on the conventional protocols, we found that during the past 20 years, $1 \mathrm{~Hz}$ stimulation protocols were most frequently used ( $n=304,22.06 \%)$, followed by $10 \mathrm{~Hz}$ $(n=230,16.70 \%), 5 \mathrm{~Hz}(n=126,9.14 \%), 20 \mathrm{~Hz}(n=122$, $8.85 \%), 25 \mathrm{~Hz}(n=22,1.60 \%)$, and $15 \mathrm{~Hz}(n=17,1.23 \%)$. See also Figure 4.

Although the stimulation intensity typically ranged from $80 \%$ to $120 \%$ of the MT, we found "popular" stimulation intensities for specific stimulation frequencies, shown in Figure 4. The substantial variability in the stimulation intensity selection approaches makes the direct comparison between the various protocols difficult. For example, the most common stimulation intensities were $90 \%$ and $110 \%$ of the MT for $1 \mathrm{~Hz}$ protocols. For the $5 \mathrm{~Hz}$ protocol, the most frequent intensity was $90 \%$ of the MT. For $10 \mathrm{~Hz}$, the most frequent intensities ranged from $90 \%$ to $110 \%$ of the MT. Note that the $10 \mathrm{~Hz}$ protocol approved by the U.S. Food and Drug Administration (FDA) uses $120 \%$ of the resting MT (George et al., 2010).

We found that the theta burst protocols ( $n=210,15.24 \%)$ used lower stimulation intensities than conventional protocols. The most common stimulation intensity was $80 \%$ of the active MT (Figure 5). Whereas for the conventional protocol, the resting MT was the most frequent intensity selection approach, for the theta burst protocols, the active MT was the dominant approach (Figure 6). Note that the U.S. FDAapproved intermittent theta burst protocol uses $120 \%$ of the resting MT (Blumberger et al., 2018).

\subsection{Reproducibility of stimulation intensity}

The seminal work of Peterchev et al. (2012) emphasized the importance of reporting all user-adjustable and devicespecific information that influences the resulting electromagnetic field. Here, we focus on the stimulation intensity from the perspective of basic science and electric field estimation approaches. We acknowledge that reproducing the dose (and not only the stimulation intensity) is a more complex and challenging task beyond the scope of this review.

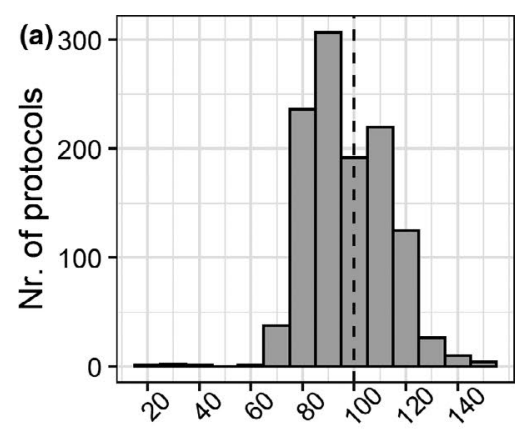

Motor threshold [percent]
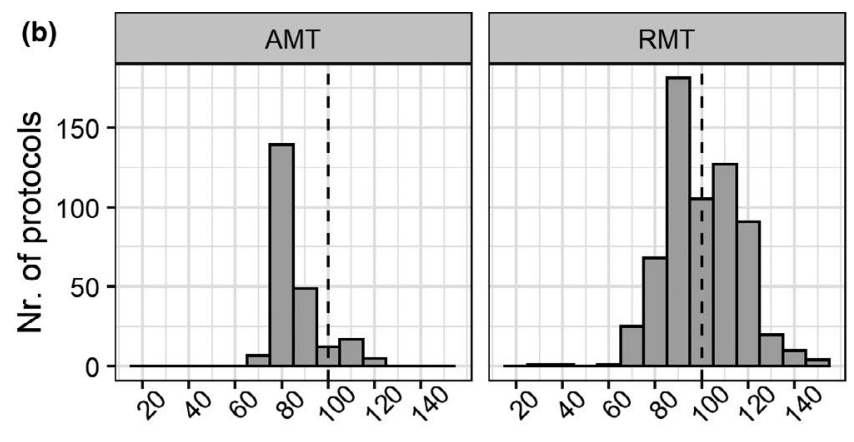

Motor threshold [percent]

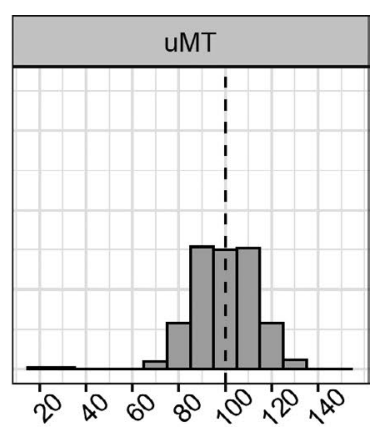

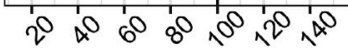

(1)

F I G U RE 3 Stimulation intensity selection based on the motor threshold approach. (a) Motor threshold percentages typically ranged from $80 \%$ to $120 \%$. Vertical dashed line highlights the intensity corresponding to the $100 \%$ motor threshold. (b) Stimulation intensity according to the type of the motor threshold. AMT indicates active motor threshold; RMT, resting motor threshold; uMT, unspecified motor threshold 

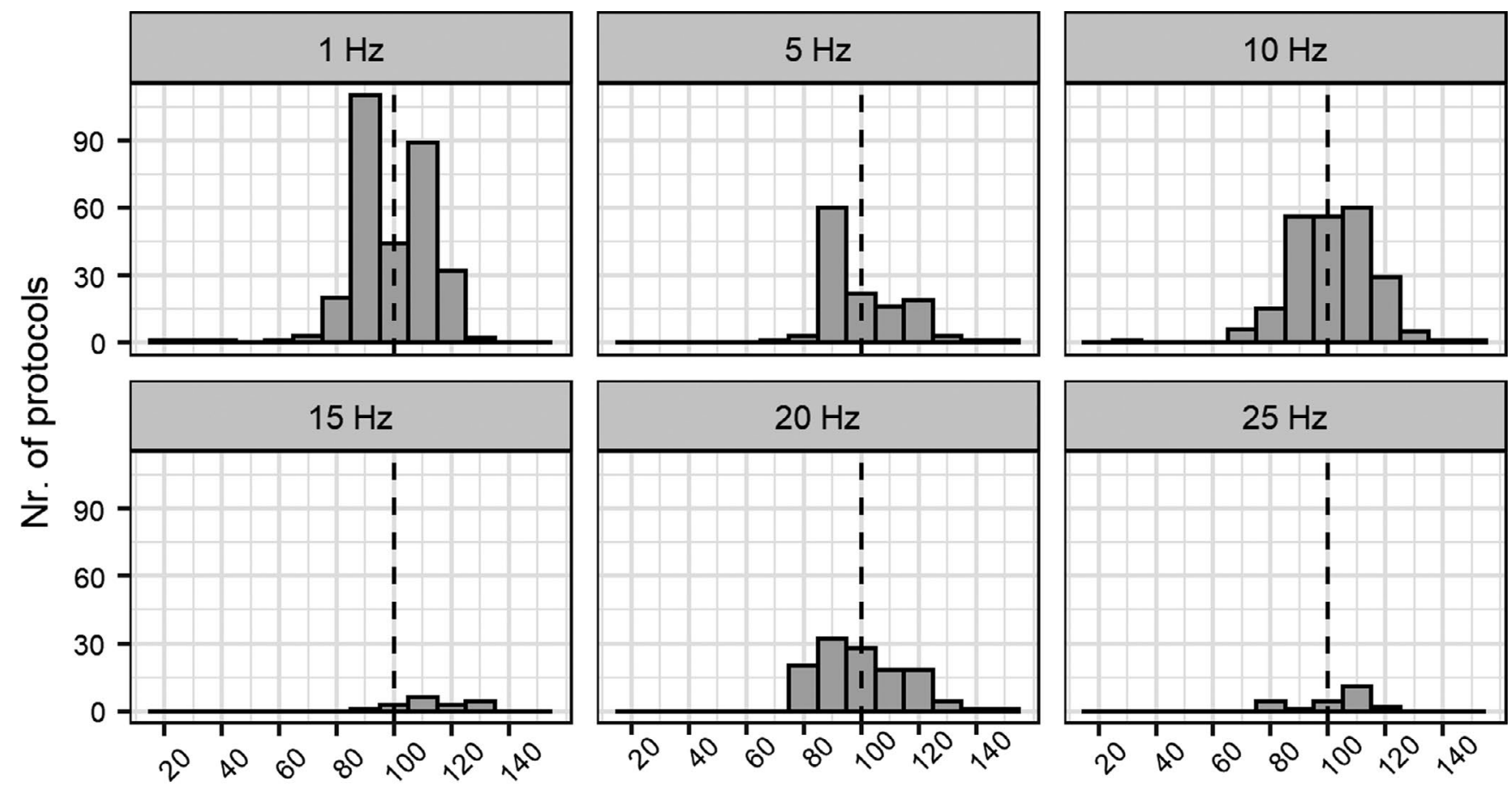

Motor threshold [percent]

F I G URE 4 Stimulation intensity expressed as a percentage of motor threshold in the conventional protocols arranged according to stimulation frequency. Note the range of intensities and differences between protocols. Intensities correspond to the resting, active, and unspecified motor thresholds

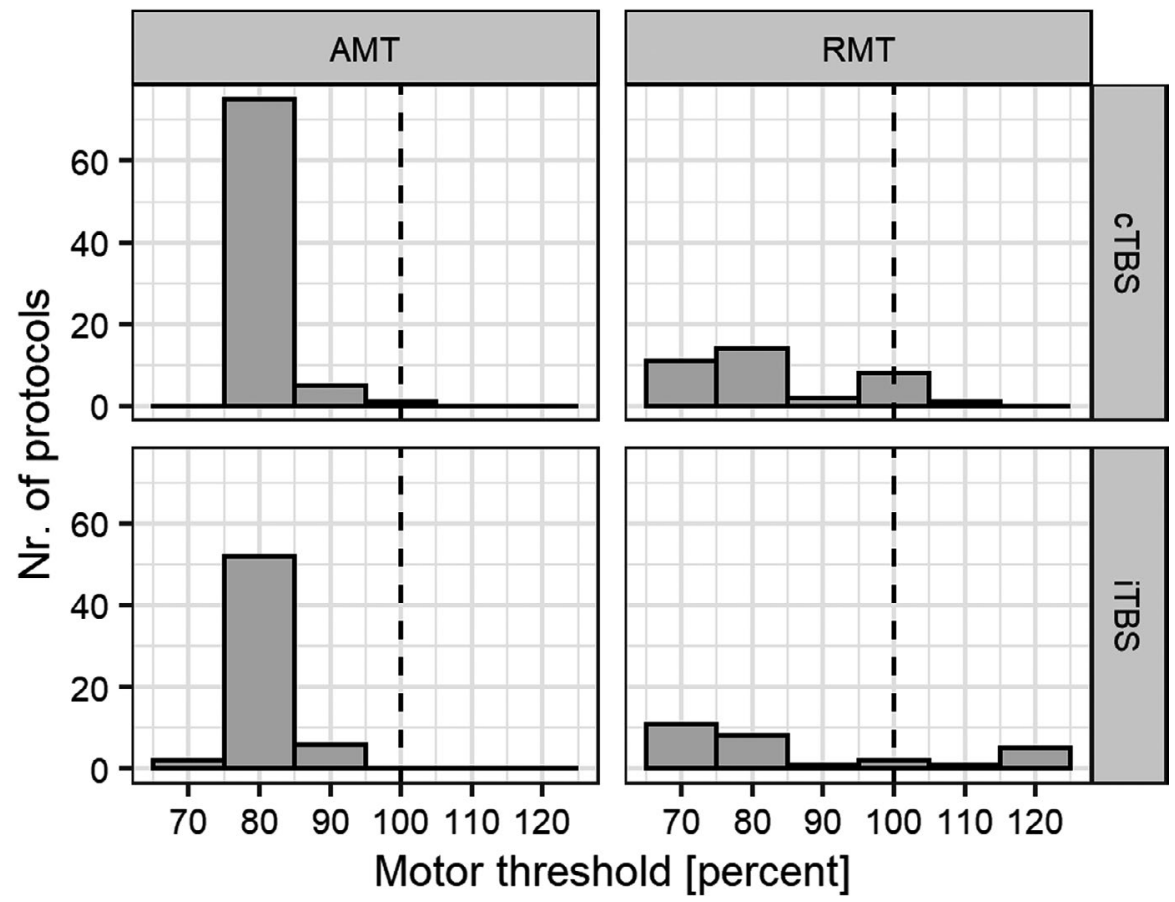

F I G URE 5 Stimulation intensity expressed as percentages of motor threshold assessed in theta burst protocols. AMT indicates active motor threshold; cTBS, continuous theta burst stimulation; iTBS, intermittent theta burst stimulation; RMT, resting motor threshold

In basic science and computational approaches, the goal is to fine-tune the stimulation parameters in a manner that closely matches the produced electromagnetic fields utilized in clinically relevant rTMS protocols. To ensure the reproducibility of the stimulation intensity, it is necessary to report (a) the individual percentages of the maximum stimulator output and (b) the company and model identification number of the rTMS device. Furthermore, in human studies, 
F I G URE 6 Relative frequency of motor threshold intensity selection approaches in conventional $(1,5$, and $10 \mathrm{~Hz})$ and theta burst protocols. AMT, active motor threshold; cTBS, continuous theta burst stimulation; FXD, fixed stimulation intensity; iTBS, intermittent theta burst stimulation; RMT, resting motor threshold; uMT, unspecified motor threshold

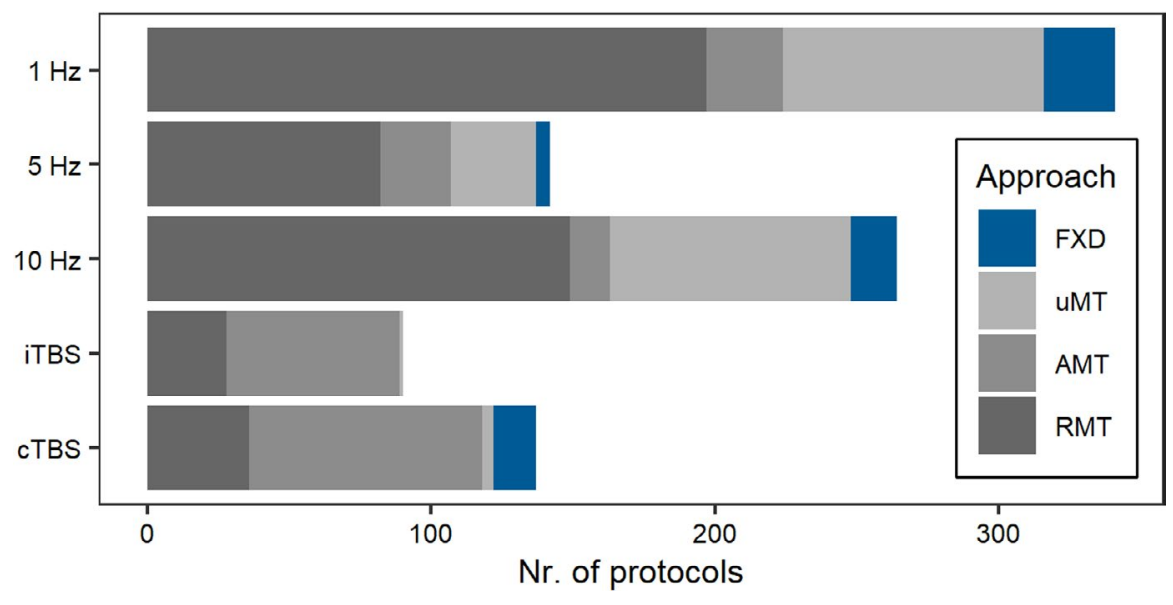

one should also describe the (c) intensity selection approach, (d) its detailed procedure, and (e) the stimulation intensity (e.g., 90\% of the resting MT). If applicable, human studies should share the defaced, anonymized, anatomical magnetic resonance imaging (MRI) data of the participants.

We determined how many studies reported all information required to reproduce the stimulation intensity (points a and b). We identified only 110 entries (7.98\%) that fulfilled these criteria.

We found that only a highly limited number of entries reported all the criteria described above (points a-e; $n=57$, $4.14 \%)$. Strictly speaking, the reported stimulation protocol is not reproducible if any of the abovementioned parameters (i.e., points a-e) are missing from the description. Strikingly, in more than half of the entries assessed here, the device output or coil model was not reported. This information is essential for approximating the induced electric fields in a given head model (Saturnino et al., 2019). Table 1 summarizes the missing information and its frequency in various year intervals.

\section{5 | Reporting electric field strength}

We identified only 39 of 3,784 studies (1.03\%) that reported the electric field strength in the brain produced by rTMS. The reported electric field values ranged from 14 to $182 \mathrm{~V} / \mathrm{m}$. Most studies reported the estimated electric field given by the neuronavigation system $(n=31,0.82 \%)$. Six used the finite element method (simulation of non-invasive brain stimulation-SimNIBS, openly available since 2015 [Thielscher et al., 2015]), one used the boundary element method, and one a spherical head model. Most studies ( $n=36,0.95 \%)$ set the stimulation intensity based on the MT and then estimated the corresponding electric field strength. We found only three studies that determined the stimulation intensity based on estimating the electric field strength.

Notably, more studies reporting the electric field strength have been published within the past 10 years, indicating an increased requirement for computational tools to estimate and standardize electric fields induced by a given rTMS protocol.

TA B LE 1 Missing information required to reproduce rTMS stimulation intensity

\begin{tabular}{|c|c|c|c|c|c|c|c|c|}
\hline Missing information & \multicolumn{4}{|c|}{ Nr. of entries } & \multicolumn{4}{|l|}{ Percent } \\
\hline Stimulation intensity & 0 & 8 & 14 & 22 & 0 & 1.39 & 2.44 & 1.60 \\
\hline Device output & 192 & 395 & 419 & 1,006 & 83.84 & 68.58 & 73.12 & 73.00 \\
\hline Coil model & 113 & 482 & 356 & 951 & 49.34 & 83.68 & 62.13 & 69.01 \\
\hline Coil model \& shape & 9 & 64 & 76 & 149 & 3.93 & 11.11 & 13.26 & 10.81 \\
\hline
\end{tabular}

Note: Examples for the missing information: intensity selection approach e.g., "active motor threshold"; stimulation intensity e.g., "90\%" of RMT; device output e.g., "50\%" of MSO.

Abbreviations: RMT, resting motor threshold; rTMS, repetitive transcranial magnetic stimulation. 


\section{4 | DISCUSSION}

In this review, we focused on how human rTMS studies determine the stimulation intensity for rTMS. To this aim, we created a database comprising 3,784 original studies and manually processed 760 randomly selected articles.

We found that the most common intensity selection approach was the MT one. Most studies used a single stimulation intensity (e.g., $110 \%$ of the resting MT). In contrast, a minority of studies used a range of stimulation intensities (e.g., $100 \%-110 \%$ of the resting MT).

The 1,10 , and $5 \mathrm{~Hz}$ were the three most popular conventional protocols. We found that most protocols used stimulation intensities from $80 \%$ to $120 \%$ of the MT. Theta burst protocols used slightly lower stimulation intensities, typically at $80 \%$ of the active MT. Whereas the threshold-based estimation approaches used individualized stimulation intensities, the less common fixed-intensity approach delivered a predetermined stimulation intensity (e.g., $50 \%$ of the device output).

According to the current reporting convention, the vast majority of articles (ca. 92\%) did not report sufficient information required to readily reproduce the stimulation intensity and approximate the electric field for rTMS. It should be noted, however, that the results of this study are based on the literature search using the PubMed engine that mainly accesses the MEDLINE database. Therefore, it is possible that we may have missed additional peer-reviewed rTMS publications that are not indexed by MEDLINE.

\section{1 | Need to link dose to electric field}

Several factors can contribute to selecting the stimulation intensity in human rTMS studies. The most frequent factors are (a) conventions of the field (e.g., using the resting MT even when targeting a different cortical region than the motor cortex itself), (b) the safety and tolerability of the participants, and (c) device limitations. Surprisingly, the electric field rarely played an explicit role in determining the stimulation intensity for rTMS (but see Beynel et al., 2020; Kraft et al., 2015; Zmeykina et al., 2020).

The variability in the electric field strength of noninvasive brain stimulation techniques significantly affects the underlying neural mechanisms (Liu et al., 2018). Electric field simulations play an essential role in linking in vitro and in vivo studies targeting cell cultures, brain slices, or the entire, in situ brains of different species (Alekseichuk et al., 2019; Lenz et al., 2016). The lack of reporting the electric field strength and participant-independent device parameters in human rTMS studies hinders the translational use of computational and basic science approaches. Mutliscale neuronal modeling is crucial in informing studies on the molecular, cellular, and neural mechanisms of rTMS (e.g., Shirinpour et al., 2020).

For instance, estimating the resting MT in rodents is different from the procedure typically used in humans due to anesthetics (c.f., Sykes et al., 2016). Similarly, it is uninformative when ex vivo studies such as brain slice experiments define the stimulation intensity exclusively using percentages of the MT (Lenz et al., 2016). In this context, reporting the corresponding device output for the stimulation intensity and the electric field is of utmost importance. Translating the MT in human participants to in vitro basic research settings is hardly possible.

However, there is substantial variability in how one can describe the resulting electric field properties. Because the produced electric field is a complex three-dimensional vector field, it is not possible to characterize it using a single parameter (Peterchev et al., 2012). Furthermore, there is an ongoing debate about which component of the rTMS-induced electric field produces the relevant physiological effects (e.g., Bungert et al., 2017; Laakso et al., 2018).

Based on these considerations, it appears to be important to share all necessary information and data required to reproduce not only the dose but also the electromagnetic field induced by a given rTMS protocol (e.g., Zmeykina et al., 2020). As the accuracy and user-friendliness of electric field simulations improve, we expect an increase in the number of studies that standardize certain electric field properties across subjects.

We tentatively suggest that studies could share at the individual level (a) the raw, anonymized, defaced, anatomical MRI (when the ethic vote and participant consent allow), (b) the exact coil location and orientation preferably saved by the neuronavigation system, (c) device characteristics, such as stimulator/coil company and model, and (d) the stimulation intensity either expressed in percentage of the maximum stimulator output and the coil current rate of change [in $\mathrm{A} / \mu \mathrm{s}]$. Furthermore, we also recommend documenting (e) the program used for the electric field simulation, (f) the coil current rate of change used for the simulation, (g) the type of head model (e.g., finite element or boundary element approach), (h) the analytical pipeline used for creating the head model, (i) the number of compartments and their conductivity values, and (j) sharing the code used for the simulation.

\section{2 | Recommendations for reproducibility}

Our analyses revealed that the overwhelming majority of studies $(92 \%)$ did not report sufficient information to reproduce the stimulation intensity. Our findings agree with the commentary of Wilson and George (2016), who draw attention to the variability and lack of methodololgical transparency and 
standardization in estimating and reporting motor-evoked potentials in human studies.

One way to overcome the limitations of the current reporting convention is to establish a consensus about standardized reporting guidelines. Editors of specialized peer-reviewed journals, who are often the leading experts in the field, can play an important role in developing and enforcing such guidelines.

At the same time, companies selling the devices should ensure access to all device-specific information (e.g., coil inductance). The model identification number should be readily available for the user to facilitate easy referencing. Thus, the authors could only report the user-adjustable parameters while referring to non-adjustable, fixed-device parameters via unique device identification numbers. The goal of such a referencing system is that authors do not have to deal with a long list of nuanced but important device-specific parameters.

Below, we list our recommendations from a basic science perspective, which may serve as an orientation for future initiatives aiming to define the relevant standards in the field (see Figure 7). We focused on the MT approach because it is unequivocally the dominant approach in the literature.

\subsection{1 | Recommendation 1}

Many studies reported using the MT approach without specifying the type of MT and the exact methods of how it was determined. Different MT approaches yield varying stimulation intensities (Siebner \& Ziemann, 2014) and electric field
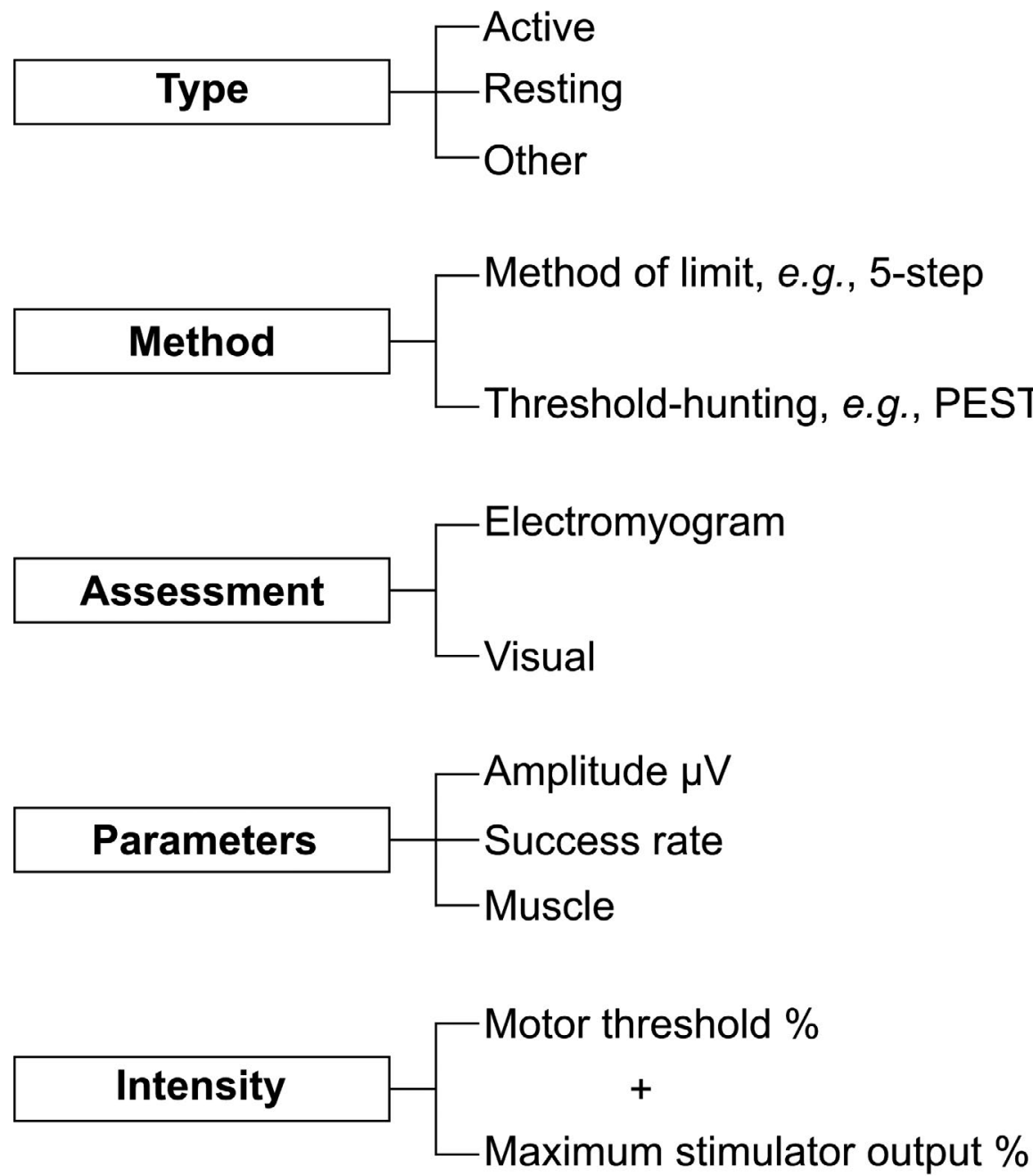

F IG URE 7 Crucial information to report when using the motor threshold intensity selection approach

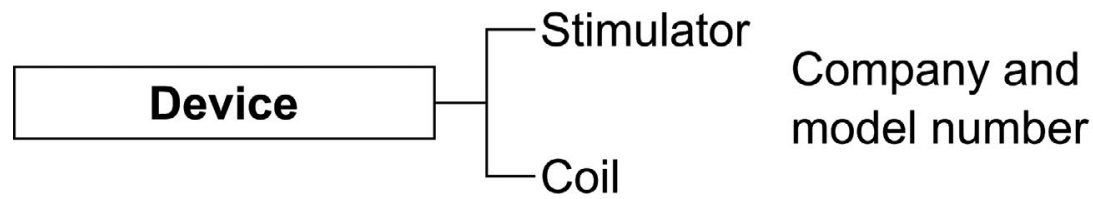


strengths. Therefore, it is essential to report explicitly about whether the MT was an active, resting MT or based on another approach, (e.g., to produce interhemispheric inhibition) (Gilio et al., 2003).

\subsection{2 | Recommendation 2}

One can estimate the threshold using various methods, such as the method of limit or threshold hunting algorithms (Awiszus, 2014; Pridmore et al., 1998; Schutter \& Honk, 2006). Many studies do not mention the method at all; instead, they only state that the MT "was established according to published criteria." Alternatively, the authors only name the method (e.g., method of limit) and refer to the procedure via citation. One obvious limitation of this reporting convention is that the article may not be available at all research institutes. Because word limitations are typically not imposed on the methods section, we recommend explicitly reporting the method's details.

\subsection{3 | Recommendation 3}

Many studies used the method of limit to estimate the active or resting MT. Although many studies used an electromyogram to record the amplitude of the motor-evoked potentials, the exact values often remained undefined. For example, the minimum amplitude for the active MT may be between 100 and $200 \mu \mathrm{V}$. A typical minimum value for the resting MT is $50 \mu \mathrm{V}$.

Another important aspect is the success rate because the exact number of successfully evoked motor potentials above a certain amplitude may vary. The typical values are 3 of 5 or 5 of 10 TMS pulses. However, we observed many other success rates. Success rates were often not reported. Therefore, we recommend reporting the peak-to-peak threshold amplitude and the success rate when using the method of limit.

\subsection{4 | Recommendation 4}

Most studies used the MT intensity selection method. Studies usually reported the stimulation intensity as a certain percentage of the MT (e.g., 90\% of the resting MT). Although describing the method of selecting the stimulation intensity is an important step, reporting the associated stimulation intensities in terms of the device output is crucial for basic science and electric field simulation approaches. For example, the active MT may correspond to $36 \%$ of the device output in one participant and $59 \%$ in another (Rounis et al., 2007). Therefore, it is essential to report the device output, typically referred to as the maximum stimulator output percentage.

\subsection{5 | Recommendation 5}

It is a common shortcoming that studies only state the manufacturer of the stimulator and the shape of the coil (e.g., figure-of-eight-shaped coil). However, the stimulator and coil parameters (e.g., coil inductance) can significantly affect the produced electromagnetic field. Different coils attached to the same stimulator may end up in different results (Lang et al., 2006). By accurately reporting the exact stimulator and coil model, one can trace these important parameters.

\section{5 | CONCLUSION}

Due to the extensive set of possible parameters, it is currently challenging to decide which parameters to report for rTMS. Consequently, the overwhelming majority of studies do not report sufficient information to reproduce the stimulation intensity and, eventually, the dose of rTMS. This failure to report stimulation parameters makes it nearly impossible to properly reproduce published protocols and design appropriate basic science experiments. There is a need for developing a standardized and easy-to-follow reporting guideline to document the most crucial stimulation parameters in the best possible way.

\section{ACKNOWLEDGMENT}

We thank Swantje Reifarth and Alina Seiler from the Department of Clinical Neurophysiology, University Medical Center Göttingen, Georg-August University, Göttingen, Germany, for their assistance in initially screening the articles. We thank Celina Jacobsen and Sarjo Kuyateh from the Institute of Psychology, UiT The Arctic University of Norway, for their assistance in manually processing the articles. We thank Lisa Kuhlmann and Anna Wolfers from the Department of Neuroanatomy, Institute of Anatomy and Cell Biology, University of Freiburg, Germany, for their assistance in manually processing the articles. The study was, in part, supported by the University Medical Center Göttingen starting grant (to ZT) and an NIH-RO1 grant (1R01NS109498-01A1 to AV). Open access funding enabled and organized by ProjektDEAL.

\section{CONFLICTS OF INTEREST}

The authors declare no conflict of interest.

\section{AUTHORS' CONTRIBUTION}

Authors' contribution was prepared according to the Contributor Roles Taxonomy. Conceptualization: ZT; Formal analysis: ZT; Funding acquisition: ZT and AV; Investigation: ZT; Methodology: ZT, MM, and AV; Project administration: ZT, WP, MM, and AV; Software: ZT; Supervision: ZT and 
AV; Visualization: ZT; Writing-original draft: ZT, ML, $\mathrm{WP}, \mathrm{MM}$, and $\mathrm{AV}$.

\section{PEER REVIEW}

The peer review history for this article is available at https:// publons.com/publon/10.1111/ejn.15195.

\section{DATA AVAILABILITY STATEMENT}

The data, the code for the literature search, and the reported analyses are available for download at the following repository: https://github.com/ZsoltTuri/2021_rTMS_inten sity_review_paper.

\section{ORCID}

Zsolt Turi (D) https://orcid.org/0000-0002-8130-7949

Matthias Mittner (D) https://orcid.org/0000-0003-0205-7353

\section{REFERENCES}

Alekseichuk, I., Mantell, K., Shirinpour, S., \& Opitz, A. (2019). Comparative modeling of transcranial magnetic and electric stimulation in mouse, monkey, and human. Neurolmage, 194, 136-148. https://doi.org/10.1016/j.neuroimage.2019.03.044

Awiszus, F. (2014). Using relative frequency estimation of transcranial magnetic stimulation motor threshold does not allow to draw any conclusions about true threshold. Clinical Neurophysiology, 125, 1285-1287. https://doi.org/10.1016/j.clinph.2013.09.046

Beynel, L., Davis, S. W., Crowell, C. A., Dannhauer, M., Lim, W., Palmer, H., Hilbig, S. A., Brito, A., Hile, C., Luber, B., Lisanby, S. H., Peterchev, A. V., Cabeza, R., \& Appelbaum, L. G. (2020). Sitespecific effects of online rTMS during a working memory task in healthy older adults. Brain Sciences, 10, 1-19.

Blumberger, D. M., Vila-Rodriguez, F., Thorpe, K. E., Feffer, K., Noda, Y., Giacobbe, P., Knyahnytska, Y., Kennedy, S. H., Lam, R. W., Daskalakis, Z. J., \& Downar, J. (2018). Effectiveness of theta burst versus high-frequency repetitive transcranial magnetic stimulation in patients with depression (THREE-D): A randomised noninferiority trial. Lancet, 391, 1683-1692. https://doi.org/10.1016/ S0140-6736(18)30295-2

Borckardt, J. J., Nahas, Z., Koola, J., \& George, M. S. (2006). Estimating Resting motor thresholds in transcranial magnetic stimulation research and practice. The Journal of ECT, 22, 169-175. https://doi. org/10.1097/01.yct.0000235923.52741.72

Bungert, A., Antunes, A., Espenhahn, S., \& Thielscher, A. (2017). Where does TMS Stimulate the motor cortex? Combining electrophysiological measurements and realistic field estimates to reveal the affected cortex position. Cerebral Cortex, 27, 5083-5094.

Chen, R., Classen, J., Gerloff, C., Celnik, P., Wassermann, E. M., Hallett, M., \& Cohen, L. G. (1997). Depression of motor cortex excitability by low-frequency transcranial magnetic stimulation. Neurology, 48 , 1398-1403. https://doi.org/10.1212/WNL.48.5.1398

George, M. S., Lisanby, S. H., Avery, D., Mcdonald, W. M., Durkalski, V., Pavlicova, M., Anderson, B., Nahas, Z., Bulow, P., Zarkowski, P., Holtzheimer, P. E., Schwartz, T., \& Sackeim, H. A. (2010). Daily left prefrontal transcranial magnetic stimulation therapy for major depressive disorder. Archives of General Psychiatry, 67, 507-516.

Gilio, F., Rizzo, V., Siebner, H. R., \& Rothwell, J. C. (2003). Effects on the right motor hand-area excitability produced by low-frequency
rTMS over human contralateral homologous cortex. Journal of Physiology, 551, 563-573.

Hamada, M., Hanajima, R., Terao, Y., Arai, N., Furubayashi, T., Inomata-Terada, S., Yugeta, A., Matsumoto, H., Shirota, Y., \& Ugawa, Y. (2007). Quadro-pulse stimulation is more effective than paired-pulse stimulation for plasticity induction of the human motor cortex. Clinical Neurophysiology, 118, 2672-2682.

Huang, Y.-Z., Edwards, M. J., Rounis, E., Bhatia, K. P., \& Rothwell, J. C. (2005). Theta burst stimulation of the human motor cortex. Neuron, 45, 201-206. https://doi.org/10.1016/j.neuron.2004.12.033

Kovalchik, S. (2017) RISmed: Download content from NCBI databases.

Kraft, A., Dyrholm, M., Kehrer, S., Kaufmann, C., Bruening, J., Kathmann, N., Bundesen, C., Irlbacher, K., \& Brandt, S. A. (2015). TMS over the right precuneus reduces the bilateral field advantage in visual short term memory capacity. Brain Stimulation, 8 , 216-223.

Laakso, I., Murakami, T., Hirata, A., \& Ugawa, Y. (2018). Where and what TMS activates: Experiments and modeling. Brain Stimulation, $11,166-174$.

Lang, N., Harms, J., Weyh, T., Lemon, R. N., Paulus, W., Rothwell, J. C., \& Siebner, H. R. (2006). Stimulus intensity and coil characteristics influence the efficacy of rTMS to suppress cortical excitability. Clinical Neurophysiology, 117, 2292-2301.

LeBeau, B. (2018). pdfsearch: Search tools for PDF files. Journal of Open Source Software, 2, 1-2.

Lenz, M., Galanis, C., Mu, F., Opitz, A., \& Wierenga, C. J. (2016). Repetitive magnetic stimulation induces plasticity of inhibitory synapses. Nature Communications, 7, 10020.

Liu, A., Vöröslakos, M., Kronberg, G., Henin, S., Krause, M. R., Huang, Y., Opitz, A., Mehta, A., Pack, C. C., Krekelberg, B., Berényi, A., Parra, L., Melloni, L., Devinsky, O., \& Buzsáki, G. (2018). Immediate neurophysiological effects of transcranial electrical stimulation. Nature Communications, 9, 5092.

Matsumoto, H., \& Ugawa, Y. (2020). Quadripulse stimulation (QPS). Experimental Brain Research, 238, 1619-1625.

Peterchev, A. V., Wagner, T. A., Miranda, P. C., Nitsche, M. A., Paulus, W., Lisanby, S. H., Pascual-leone, A., \& Carolina, N. (2012). Fundamentals of transcranial electric and magnetic stimulation dose: Definition, selection, and reporting practices. Brain Stimulation, 5, 435-453. https://doi.org/10.1016/j. brs.2011.10.001

Pridmore, S., Fernandes Filho, J. A., Nahas, Z., Liberatos, C., \& George, M. S. (1998). Motor threshold in transcranial magnetic stimulation: A comparison of a neurophysiological method and a visualization of movement method. The Journal of ECT, 14, 25-27. https://doi. org/10.1097/00124509-199803000-00004

R Core Team (2020). $R$ : A language and environment for statistical computing. Vienna, Austria: R Foundation for Statistical Computing. http://www.R-project.org/

Rounis, E., Yarrow, K., \& Rothwell, J. C. (2007). Effects of rTMS conditioning over the fronto-parietal network on motor versus visual attention. Journal of Cognitive Neuroscience, 19, 513-524.

RStudio Team (2020). RStudio: Integrated development for R. Boston, MA: RStudio, PBC. http://www.rstudio.com/.

Saturnino, G. B., Puonti, O., Nielsen, J. D., Antonenko, D., Madsen, K. H., \& Thielscher, A. (2019) SimNIBS 2.1: A comprehensive pipeline for individualized electric field modelling for transcranial brain stimulation. In S. Makarov, M. Horner \& G. Noetscher (Eds.), Brain and human body modeling (pp. 3-25). cham: Springer. https:// doi.org/10.1007/978-3-030-21293-3_1 
Schutter, D. J. L. G., \& Honk, J. V. (2006). A standardized motor threshold estimation procedure for transcranial magnetic stimulation research. The Journal of ECT, 22, 176-178. https://doi. org/10.1097/01.yct.0000235924.60364.27

Shirinpour, S., Hananeia, N., Rosado, J., Galanis, C., Vlachos, A., Jedlicka, P., Queisser, G., \& Opitz, A. (2020) Multi-scale modeling toolbox for single neuron and subcellular activity under (repetitive) transcranial magnetic stimulation. bioRxiv, 2020.09.23.310219.

Siebner, H. R., \& Ziemann, U. (2014). What is the threshold for developing and applying optimized procedures to determine the corticomotor threshold? Clinical Neurophysiology, 125, 1-2.

Suppa, A., Huang, Y. Z., Funke, K., Ridding, M. C., Cheeran, B., Di Lazzaro, V., Ziemann, U., \& Rothwell, J. C. (2016). Ten years of theta burst stimulation in humans: Established knowledge, unknowns and prospects. Brain Stimulation, 9, 323-335. https://doi. org/10.1016/j.brs.2016.01.006

Sykes, M., Matheson, N. A., Brownjohn, P. W., Tang, A. D., Rodger, J., Shemmeii, J. B. H., \& Reynolds, J. N. J. (2016). Differences in motor evoked potentials induced in rats by transcranial magnetic stimulation under two separate anesthetics: Implications for plasticity studies. Frontiers in Neural Circuits, 10, 80.

Thielscher, A., Antunes, A., \& Saturnino, G. B. (2015). Field modeling for transcranial magnetic stimulation: A useful tool to understand the physiological effects of TMS? Annual International Conference of the IEEE Engineering in Medicine and Biology Society, 2015, $222-225$.

Wilson, M. T., \& George, L. S. (2016). Repetitive Transcranial magnetic stimulation: A call for better data. Frontiers in Neural Circuits, 10, 57.

Zmeykina, E., Mittner, M., Paulus, W., \& Turi, Z. (2020). Weak rTMSinduced electric fields produce neural entrainment in humans. Scientific Reports, 10, 1-16.

\section{SUPPORTING INFORMATION}

Additional supporting information may be found online in the Supporting Information section.

How to cite this article: Turi Z, Lenz M, Paulus W, Mittner M, Vlachos A. Selecting stimulation intensity in repetitive transcranial magnetic stimulation studies: A systematic review between 1991 and 2020. Eur J Neurosci. 2021;00:1-12. https://doi.org/10.1111/ ejn.15195 